\title{
Proposta de Modelagem Híbrida PROMETHEE-SAPEVO-M1: avalia- ção multicritério de drones para emprego na guerra naval
}

\author{
Miguel Ângelo Lellis Moreira \\ Instituto Militar de Engenharia (IME) \\ Praça Gen. Tibúrcio, 80 - Urca, Rio de Janeiro - RJ, 22290-270 miguellellis@hotmail.- \\ com \\ Marcos dos Santos \\ Instituto Militar de Engenharia (IME) \\ Praça Gen. Tibúrcio, 80 - Urca, Rio de Janeiro - RJ, 22290-270 marcosdossantos_dou- \\ torado_uff@yahoo.com.br \\ Carlos Francisco Simões Gomes \\ Universidade Federal Fluminense (UFF) \\ Rua Passos da Pátria, 156, Bloco D, São Domingos, Niterói - RJ, 24210-240 cfsg1@bol.- \\ com.br
}

\begin{abstract}
RESUMO
0 presente trabalho objetiva apresentar um estudo de caso baseado na necessidade da Marinha do Brasil em realizar uma possível aquisição de RPAS (Remotely Piloted Aircraft System - Sistema de Aeronave Remotamente Pilotada) para emprego na guerra naval. Para análise decisória do caso, é apresentado uma nova modelagem híbrida (PROMETHEE-SAPEVO-M1), baseada nos métodos de Apoio Multicritério à Decisão, que viabiliza uma avaliação com dados qualitativos e quantitativos no dado caso. Além do método proposto, foi desenvolvido uma ferramenta computacional em linguagem Python, destinado a auxiliar o agente decisor no processo de análise e avaliação das aeronaves quanto aos critérios requeridos. 0 método proposto expõe sua eficácia, a partir do momento em que permite a avaliação de dados com diferentes naturezas em um formato equivalente de avaliação, disponibilizando a Marinha do Brasil uma avaliação completa em relação as aeronaves remotamente pilotadas mais favoráveis ao emprego na guerra naval.
\end{abstract}

Palavra-chave: Drones; Sistema de Aeronave Remotamente Pilotada; PROMETHEE; SAPEVO-M; Apoio Multicritério à Decisão; Ferramenta Computacional. 
This paper aims to present a case study based on the need of the Brazilian Navy to make a possible acquisition of RPAS (Remotely Piloted Aircraft System) for use in naval warfare. For the decision analysis of this case, a new hybrid modeling (PROMETHEE-SAPEVO-M1) is presented, based on the Multicriteria Decision Aid Methods, which enables an evaluation with qualitative and quantitative data in the case. In addition to the proposed method, a computational tool developed in Python language, was used to assist the decision-maker agent in the aircraft analysis and evaluation process as to the required criteria. The proposed method proves to be effective, since it allows the evaluation of data with different natures in an equivalent evaluation format, providing the Brazilian Navy with a complete evaluation to the most favorable remotely piloted aircraft in naval warfare.

Keywords: Drones; Remotely Piloted Aircraft System; PROMETHEE; SAPEVO-M; Multicriteria Decision Aiding; Computational Tool.

\section{Como Citar:}

MOREIRA, Miguel Ângelo Lellis; SANTOS, Marcos dos; GOMES, Carlos Francisco Simões. Proposta de Modelagem Híbrida PROMETHEE-SAPEVO-M1: avaliação multicritério de drones para emprego na guerra naval. In: SIMPÓSIO DE PESQUISA OPERACIONAL E LOGÍSTICA DA MARINHA, 19., 2019, Rio de Janeiro, RJ. Anais [...]. Rio de Janeiro: Centro de Análises de Sistemas Navais, 2019.

\section{INTRODUÇÃO}

A ARP (Aeronave Remotamente Pilotada), também conhecida como "drone", vem despertando interesse nos últimos anos por suas inúmeras e variadas aplicações. Inicialmente sendo considerada apenas como um aparelho voador controlado a distância e com recursos limitados, atualmente as ARPs apresentam em seus sistemas, recursos e tecnologias mais elaboradas, viabilizando seu emprego em âmbito civil e militar, principalmente nas operações de guerra naval (BRAGA, 2019).

Com o avanço tecnológico das últimas décadas, permitiu-se o aprimoramento desses equipamentos. A inclusão de dispositivos tecnológicos, como sensores, radares, câmeras e até armamento, em alguns casos específicos, proporcionou a expansão de suas aplicações. Conforme Braga (2019) expõem, nas ações de guerra naval, hoje as ARPs são empregadas em operações de logística, vigilância, sensoriamento, reconhecimento e apoio ao combate.

O emprego das ARPs nas operações navais comporta variados tipos de aplicações, assim sendo define-se guerra naval na Marinha do Brasil: "Parte constituída por ações militares conduzidas nos espações marítimos, nas águas interiores e em certas áreas terrestres limitadas de interesse para as operações navais, incluindo o espaço aéreo sobrejacente. Consiste no emprego do Poder Naval, contribuindo para a conquista e a manutenção dos Objetivos Nacionais de Defesa" (BRASIL, 2014)

Conforme apresenta (SILVA, 2016), uma das principais características dos drones é a ausência de um piloto embarcado e algumas das suas vantagens são o equipamento viabilizar voos em baixas altitudes e espaços restritos, minimizando sua visibilidade, permitir transmissão de imagens e vídeos em tempo real e a capacidade de customização e convergência de tecnologias. Segundo Floreano e Wood (2015), é esperado este tipo de tecnologia apresente um grande impacto em atividades na 
sociedade, incluindo transporte, comunicação, agricultura, mitigação de desastres e preservação do meio ambiente.

Inúmeros tem sido os modelos de equipamentos desenvolvidos por empresas de renome tecnológico, com as mais variadas especificações de sistema (GIONES e BREM, 2017). Para a aplicação de uma ARP, deve-se considerar um sistema como um todo, assim um RPAS (Remotely Piloted Aircraft System - Sistema de Aeronave Remotamente Pilotada) é composto por quatro subsistemas, são eles: A aeronave, carga útil (sensores e armamento), estação de controle e equipamentos de comunicação (PECHARROMÁN e VEIGA, 2016).

Segundo Braga (2019), para o emprego das ARPs na guerra naval, deve-se considerar um conjunto de critérios e variáveis que influenciam de forma direta na escolha do sistema mais favorável, alguns deles são: velocidade, alcance, peso, autonomia, altitude, tipos de equipamentos entre outros. Considerando a presença de trade-off entre os critérios de avaliação, a estruturação do problema junto a aplicação de métodos de Apoio Multicritério à Decisão (AMD), viabilizariam a Marinha do Brasil, uma análise robusta quanto a análise decisória relacionada a qual ARP adquirir para seu emprego em suas operações navais.

Para Gomes e Gomes (2019), os métodos AMD, podem ser compreendidos como técnicas que viabilizam ao decisor (seja indivíduo, grupos ou organizações) a estruturação e análise de problemas complexos de avaliação de forma transparente, com a introdução de critérios quantitativos e qualitativos, em casos específicos, havendo trade-off entre eles. Esses métodos permitem aos agentes decisores estruturarem um processo decisório, considerando vários aspectos de avaliação, tais como técnicos, socioeconômicos e ambientais por exemplo, em níveis operacionais e estratégicos para tomada de decisão (GRECO; FIGUEIRA; EHRGOTT, 2016).

Relacionado a uma análise de decisão para dados quantitativos, o método da escola francesa PROMETHEE (Preference Ranking Organization Method for Enrichment Evaluations) está relacionado aos modelos de relações hierárquicas, em que a ordem preferencial da variável de cada critério analisado é normalizada por uma função de preferência. 0 decisor tem por função indicar informações entre cada critério, no caso de quem deve ser maximizado ou minimizado, e qual funções de preferência a ser empregada para cada caso (BRANS e SMET, 2016).

Viabilizando uma análise por dados qualitativos com entradas verbais, tem-se o método SAPEVO-M (Simple Aggregation of Preferences Expressed by Ordinal Vectors - Multi Decision Makers), evolução do método original SAPEVO (GOMES; MURY; GOMES, 1997), destinado para problemáticas que visam esclarecer a decisão pelo reagrupamento das ações em classes de equivalência, ordenando parcialmente ou de modo completo, segundo as preferências do(s) decisor(es).

0 presente trabalho será apresentado em uma estrutura de estudo de caso e tem como propósito estar aplicando uma modelagem híbrida composta pelos métodos PROMETHEE e SAPEVO-M, viabilizando uma análise decisória composta por dados de diferentes naturezas. Também será apresentado uma aplicação desenvolvida em Python 3.7 com o objetivo de auxiliar na implementação do método proposto no dado caso. A nova modelagem terá por objetivo auxiliar a Marinha do Brasil no processo de análise decisória quanto a uma possível aquisição de um sistema ARP para o emprego na guerra naval. 


\section{ARP (AERONAVE REMOTAMENTE PILOTADA)}

0 termo "drone" obteve sua origem nos Estados Unidos da América em meados dos anos 1970, proporcionando inúmeras vantagens para as operações militares, sendo inicialmente usados para reunir informações em missões de reconhecimento ou atividades que envolviam alto risco (GIONES e BREM, 2017). No brasil, o termo é mais designado para dispositivos de proporções pequenas, destinados a lazer e filmagens aéreas (PECHARROMÁN e VEIGA, 2016).

Segundo Silva (2018), no Brasil, o termo Aeronave Remotamente Pilotada (ARP) é o mais adequado tratando-se de aplicações militares e comerciais. Uma ARP é caracterizada por ser uma aeronave não tripulada, porém controlada remotamente por um piloto, por meio de uma interface externa, podendo ser: computador, dispositivo digital, sistema de controle etc.

Considerando que a tecnologia, para seu funcionamento, é composta por outros dispositivos, um Sistema de Aeronave Remotamente Pilotada deve ser considerado. Um RPAS é composto por um conjunto de ARPs, uma estação de controle (Ground Control Station - GSC), dispositivos de comunicação e equipamentos classificados como carga útil, destinados a obtenção de dados e informações ou apoio a operações. Buscando amplo entendimento, o termo SARP, tradução de RPAS, não é mais utilizado, por já haver aplicação do termo, que é acrônimo de Standard and Recommended Procedures, e o mesmo ser utilizado internacionalmente para orientações emitidas ao segmente aeronáutico (BRASIL, 2018).

\section{MODELAGEM HÍBRIDA PROMETHEE-SAPEVO-M1}

O novo método é caracterizado por uma modelagem que tem por objetivo "unir" dois métodos de análise multicriterial, são eles: PROMETHEE (BRANS e VINCKE, 1985) e SAPEVO-M, evolução do método SAPEVO (GOMES; MURY; GOMES, 1997). A modelagem é baseada na estrutura básica do método PROMETHEE, entretanto este é destinado a problemáticas onde há dados numéricos, logo as técnicas presentes no método SAPEVO-M objetivam habilitar a nova modelagem uma avaliação com dados de natureza qualitativa.

A proposta também viabiliza ao decisor, definir pesos para os critérios de avaliação, por meio de técnicas de comparação presentes no SAPEVO-M, porém este é destinado a aplicações onde há múltiplos decisores. Considerando que o método proposto é destinado a avaliações onde há apenas um agente decisor, a avaliação de ponderação é apresentada com algumas adaptações quanto sua versão base.

\subsection{FUNCIONALIDADE DO MÉTODO}

0 método é trabalhado em etapas, sendo a primeira destinada a estruturação do problema, definindo o número de alternativas ou ações para avaliação, os critérios de caráter qualitativo e quantitativo. Na sequência as alternativas serão primeiramente avaliadas quanto aos critérios de natureza qualitativa e logo após analisadas quanto aos critérios quantitativos. Esta última é trabalhada conforme a estrutura base do método PROMETHEE.

As etapas seguintes são destinadas a aplicação dos pesos para seus respectivos critérios e obtenção dos fluxos de preferência global e análises dos resultados. 0 processo lógico matemático presente em cada etapa será exposto nos tópicos seguintes. 


\subsubsection{Estruturação do Problema}

0 processo de avaliação é estruturado em forma de uma matriz (critérios $\mathrm{x}$ alternativas), considerando um conjunto de alternativas $A$, onde $\mathrm{a}_{\mathrm{i}} \in \mathrm{A}, \mathrm{i}=1, \ldots \mathrm{n}$, sendo avaliadas quanto a um conjunto de critérios qualitativos $h, h=1, \ldots L$, e um conjunto de critérios quantitativos $j, j=1, \ldots \mathrm{k}$.

\begin{tabular}{|c|c|c|c|c|c|}
\hline \multirow{2}{*}{ Critérios } & \multicolumn{5}{|c|}{ Alternativas } \\
\hline & $\mathbf{a}_{1}$ & $\mathbf{a}_{2}$ & $\mathbf{a}_{3}$ & ... & $a_{n}$ \\
\hline $\mathbf{h}_{1}$ & $a_{11}$ & $a_{21}$ & $a_{31}$ & $\ldots$ & $a_{n 1}$ \\
\hline $\mathbf{h}_{2}$ & $a_{12}$ & $a_{22}$ & $a_{32}$ & $\ldots$ & $a_{n 2}$ \\
\hline $\mathbf{h}_{\mathbf{L}}$ & $a_{1 L}$ & $a_{2 L}$ & $a_{3 L}$ & $\ldots$ & $a_{n L}$ \\
\hline $\mathbf{j}_{1}$ & $a_{11}$ & $a_{21}$ & $a_{31}$ & $\ldots$ & $a_{n 1}$ \\
\hline $\mathbf{j}_{2}$ & $a_{12}$ & $a_{22}$ & $a_{32}$ & $\ldots$ & $a_{\mathrm{n} 2}$ \\
\hline $\mathbf{j}_{\mathbf{k}}$ & $a_{1 k}$ & $a_{2 k}$ & $a_{3 k}$ & $\ldots$ & $a_{n K}$ \\
\hline
\end{tabular}

\subsubsection{Avaliação Quanto aos Critérios Qualitativos}

A avaliação será realizada quanto aos critérios de natureza qualitativa $h, h=$ $1, \ldots L .0$ processo é caracterizado por uma análise comparativa entre as alternativas pertencentes ao conjunto A, para cada critério pertencente ao conjunto $h$. A análise é baseada em uma avaliação de preferência presente em uma escala de importância, tabela 1.

Tabela 1 - Escala de Importância.

\begin{tabular}{|l|c|}
\hline Absolutamente pior / Absolutamente menos importante & $\mathbf{- 3}$ \\
\hline Muito Pior / Muito menos importante & $\mathbf{- 2}$ \\
\hline Pior / Menos importante & $\mathbf{- 1}$ \\
\hline Equivalente / Tão importante quanto & $\mathbf{0}$ \\
\hline Melhor / Mais importante & $\mathbf{1}$ \\
\hline Muito melhor / Muito mais importante & $\mathbf{2}$ \\
\hline Absolutamente melhor / Absolutamente mais importante & $\mathbf{3}$ \\
\hline
\end{tabular}

Para cada critério qualitativo avaliado, é obtido uma matriz de comparação entre os elementos pertencentes ao conjunto A. Por meio da equação (2) os valores são normalizados, obtendo um grau de importância daquela alternativa no dado critério.

$$
v=\frac{a_{i j}-\min a_{i j}}{\max a_{i j}-\min a_{i j}}
$$

Obtido os valores de importância das alternativas no critério avaliado, as

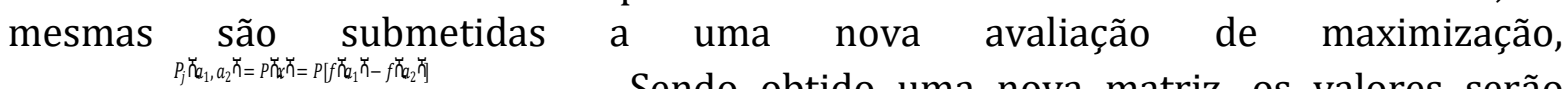
normalizados por uma função com variação linear.

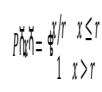

Ao final é obtido uma matriz normalizada para cada critério pertencente ao conjunto $h$. 


\subsubsection{Avaliação Quanto aos Critérios Quantitativos}

Avaliação das alternativas quanto ao conjunto de critério quantitativos $j, j=$ $1, \ldots$ k. Nesta etapa é mantido a estrutura de análise pertencente ao método PROMETHEE em sua modelagem básica.

Para cada critério definido é necessário especificar uma função de preferência generalizada $\left(\mathrm{P}_{\mathrm{j}}\right)$ tal que:

$$
\mathrm{Pj}: \mathrm{A} \times \mathrm{A} \rightarrow[0,1]
$$

Comparando as alternativas $a_{1}$ e $a_{2}$, pertencentes ao conjunto $A$, tem-se:

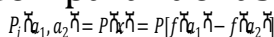
, representando o grau de preferência de $a_{1}$ sobre $a_{2}$ segundo o critério $j$.

Quando o critério precisa ser maximizado, usa-se $\mathrm{x}=f\left(\mathrm{a}_{1}\right)-f\left(\mathrm{a}_{2}\right)$ para definição da função de preferência. Caso haja a necessidade de minimização do critério, a função de preferência será dada por $\mathrm{x}=f\left(\mathrm{a}_{2}\right)-f\left(\mathrm{a}_{1}\right)$.

De acordo com Brans, Vincke e Mareschal (1986) há seis tipos de funções de preferência generalizadas, não sendo exaustivo a essas, porém satisfazem a maioria dos casos de aplicações práticas. As funções são definidas na tabela a seguir:

Tabela 2 - Funções de Preferência.

\begin{tabular}{|c|c|c|}
\hline Tipo & $P(x)$ & Parâmetros fixados \\
\hline Usual & 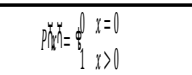 & - \\
\hline U-Shape & $M=\left\{\begin{array}{l}r \leq n \\
x>n\end{array}\right.$ & $Q$ \\
\hline V-Shape & 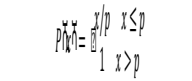 & $P$ \\
\hline Level & 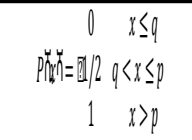 & $q, p$ \\
\hline V-Shape I & 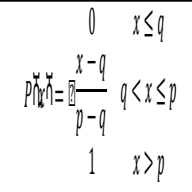 & $q, p$ \\
\hline Gaussian & 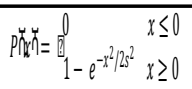 & $s$ \\
\hline
\end{tabular}

Fonte: (BRANS; VINCKE; MARESCHAL, 1986).

- Na função de preferência Usual há indiferença entre as alternativas $a_{1}$ e $a_{2}$ se . Quando as avaliações são diferentes o avaliador tem preferência total pela alternativa de melhor avaliação.

- Na função de preferência U-Shape, as alternativas serão indiferentes, caso não excedam o limite de indiferença $q$. Caso contrário, será dada preferência pela alternativa com melhor avaliação. 
- A função de preferência $V$-Shape, $p$ é o limite de preferência e, se a diferença entre as alternativas for menor que esse limite, a preferência pela alternativa $a_{1}$ quanto $a_{2}$ aumenta linearmente com a diferença $x$. Quando a diferença é maior que $p$, há total preferência pela alternativa $a_{1}$.

- Na função de preferência Level, o avaliador define o limite de indiferença q e o limite de preferência $p$, tal que: sendo x menor que q, haverá indiferença entre as alternativas. Se x está entre q e p, há fraca preferência por a1 quanto $\mathrm{a}_{2}$, e se x é maior que $\mathrm{p}$, $\mathrm{a}_{1}$ é preferível à $\mathrm{a}_{2}$.

- Na função de preferência V-Shape I o avaliador considera que sua preferência aumenta linearmente da indiferença para a preferência entre os limites $q$ e $p$.

- A função de preferência Gaussian é necessário ao decisor definir o parâmetro $s$, responsável por indicar o valor a partir do qual há mudança na concavidade na curva de preferência.

Ao final é obtido as matrizes normalizadas, sendo avaliadas em conjunto com as informações da etapa anterior nas fases seguintes.

\subsubsection{Definição de Pesos}

Considerando uma análise monodecisor, a avaliação SAPEVO-M1 irá considerar os critérios qualitativos e quantitativos em um só conjunto j, sendo j $=1, \ldots$, $L+k$. Considerando a escala de importância abaixo, será obtido o quanto determinado critério é importante em relação aos demais do conjunto.

Tabela 3 - Escala de Importância.

\begin{tabular}{|l|c|}
\hline Absolutamente pior / Absolutamente menos importante & $\mathbf{- 3}$ \\
\hline Muito Pior / Muito menos importante & $\mathbf{- 2}$ \\
\hline Pior / Menos importante & $\mathbf{- 1}$ \\
\hline Equivalente / Tão importante quanto & $\mathbf{0}$ \\
\hline Melhor / Mais importante & $\mathbf{1}$ \\
\hline Muito melhor / Muito mais importante & $\mathbf{2}$ \\
\hline Absolutamente melhor / Absolutamente mais importante & $\mathbf{3}$ \\
\hline
\end{tabular}

No SAPEVO-M1 é considerado um valor de soma máxima e mínima possível dentro do conjunto de critérios j.

A soma máxima é obtida por $x=(n-1)$. 3, representando o maior valo de soma possível dentro daquela avaliação. Quanto mais próximo um critério estiver deste valor, maior será sua dominância no conjunto.

A soma mínima é obtida por $x=(n-1) \cdot-3$, representando o menor valor de soma possível dentro da avaliação. Quanto menor o valor de um critério e mais próximo da soma mínima este estiver, o critério apresentará pouca importância no conjunto.

Os valores obtidos na avaliação serão normalizados pela equação (5). Após a normalização, o somatório dos graus obtidos será equivalente a 1 e os pesos serão ponderados quantos aos seus respectivos critérios.

$$
v=\frac{a_{i j}-(\text { soma mínima })}{\text { (soma máxima) }- \text { (soma mínima) }}
$$




\subsection{5. Índice de Preferência Ponderada Global}

Com as matrizes normalizadas e os pesos obtidos, será calculado para cada par comparado, o índice de preferência ponderada global $\pi\left(a_{1}, a_{2}\right)$, indicando o percentual de preferência da alternativa $a_{1}$ em relação a alternativa $a_{2}$, sendo considerado os pesos atribuídos a cada critério definido.

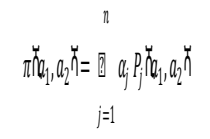

Onde:

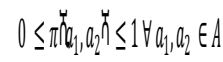

\subsubsection{Fluxo de Importância Positivo}

É caracterizado pela representação da média de preferência de $\mathrm{a}_{1}$ sobre todas as demais alternativas pertencentes ao conjunto $\mathrm{A}$.

$$
\phi^{+}\left(a_{1}\right)=\frac{1}{n-1} \prod_{n \in A}^{n} \pi n_{Q_{1}, 1}^{n}
$$

Assim sendo, quanto maior for o fluxo de importância positivo para $\mathrm{a}_{1} \mathrm{C}$ Jmelhor será a alternativa.

\subsubsection{Fluxo de Importância Negativo}

Ao contrário do modelo apresentado no item anterior, o fluxo de importância negativo é representado pela média de preferência de todas as alternativas sobre $\mathrm{a}_{1}$.

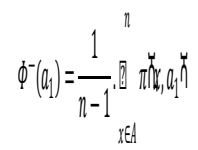

Quanto menor for o fluxo negativo para $\mathrm{a}_{1} \mathrm{C}$

), melhor será a alternativa.

\subsubsection{Fluxo de Importância Líquido}

Definido os fluxos de importância positivo e negativo, será definido o fluxo líquido, com objetivo de gerar os graus de importância para cada alternativa e hierarquiza-las seguindo uma ordem decrescente. Este fluxo é calculado pela diferença encontrada entre os fluxos positivo e negativo, conforme é apresentado a seguir:

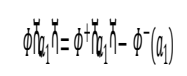

- Onde $\mathrm{a}_{1}$ é preferível à $\mathrm{a}_{2}$

- Onde $\mathrm{a}_{1}$ é indiferente à $\mathrm{a}_{2}$

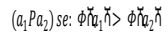

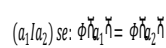




\subsection{AVALIAÇÃO DE PREFERÊNCIA PARCIAL}

Baseada no modelo de avaliação do método PROMETHEE I, é feito uma análise de pré-ordem parcial das alternativas, sendo obtida por meio dos fluxos de importância positivo e negativo. Normalmente, ambos os fluxos não obtêm os mesmos resultados, logo este método de análise é compreendido como o cruzamento entre os fluxos (BRANS e VINCKE, 1985).

Para ser obtido a pré-ordem parcial das alternativas é definia as relações de preferência (P), indiferença (I) e incompatibilidade (R).

- Preferência $\mathrm{a}_{1} \mathrm{~Pa}_{2}$

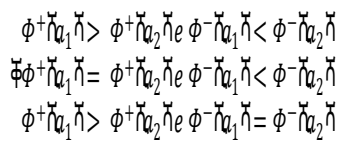

- Indiferença $\mathrm{a}_{1} I \mathrm{a}_{2}$

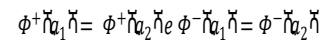

- Incompatibilidade $\mathrm{a}_{1} R \mathrm{a}_{2}$

Na relação de preferência, uma força da alternativa $a_{1}$ está associada a uma pequena fraqueza de $\mathrm{a}_{1}$ relacionada $\mathrm{a} \mathrm{a}_{2}$, logo, é considerado que ambos os fluxos, positivo e negativo, são consistentes e podem ser considerados como verdades.

Para a relação de igualdade, ambos os fluxos devem ser iguais para a as alternativas em comparação.

Para que a relação de incompatibilidade seja considerada, é necessário que uma força de $a_{1}$ esteja relacionada a uma fraqueza de $a_{2}$, isso normalmente ocorre quando $\mathrm{a}_{1}$ é bom em um conjunto de critérios que $\mathrm{a}_{2}$ é fraco.

\subsection{AVALIAÇÂO DE PREFERÊNCIA TOTAL}

Modelo de análise presente no método PROMETHEE II, este consiste na utilização das relações de preferência (P) e indiferença (I), utilizando o fluxo líquido de importância obtido pela equação (10), viabilizando gerar uma pré-ordem completa. Conforme apresentam Brans, Vincke e Mareschal (1986), o fluxo líquido pode ser reconhecido como o equilíbrio entre os fluxos positivos e negativos, logo, quanto maior o valor obtido, melhor a alternativa de escolha.

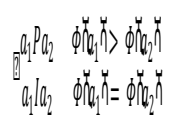

Com a obtenção das relações de preferência, a ordenação será construída de forma decrescente quanto aos valores obtidos no fluxo de importância líquido respectivo a cada alternativa. 


\subsection{AVALIAÇÂO DE PREFERÊNCIA POR INTERVALOS}

Conforme é apresentado por Tzeng e Huang (2011), a avaliação de preferência por intervalos, modelo de análise pertencente ao método PROMETHEE III, associa para cada alternativa a1, um intervalo $\left[x\left(\mathrm{a}_{1}\right), y\left(\mathrm{a}_{1}\right)\right]$, definindo uma pré-ordem completa dos intervalos $(P, I)$, apresentado na sequência:

$$
\begin{gathered}
a_{1} P a_{2} \text { se } x_{a_{1}}>y_{a_{2}} \\
a_{1} I a_{2} \text { se } x_{a_{1}} \leq y_{a_{2}} \text { e } x_{a_{2}} \leq y_{a_{1}}
\end{gathered}
$$

0 intervalo $\left[x\left(\mathrm{a}_{1}\right), y\left(\mathrm{a}_{1}\right)\right]$, é dado por:

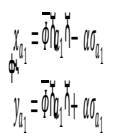

Onde n é reconhecido como o número de critérios:

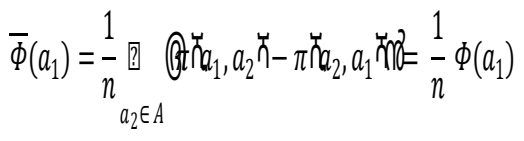

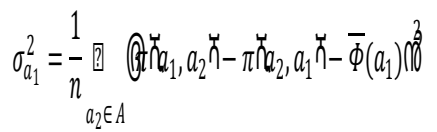

Simplificando $\left[x\left(\mathrm{a}_{1}\right), y\left(\mathrm{a}_{1}\right)\right]$, é um intervalo onde o centro é o fluxo médio líquido de a1 e o comprimento do qual é proporcional ao erro padrão da distribuição dos números $\pi\left(\mathrm{a}_{1}, \mathrm{a}_{2}\right)-\pi\left(\mathrm{a}_{2}, \mathrm{a}_{1}\right)$. Ainda, quanto menor o valor de $\mathrm{a}_{1}$, maior será o valor de superação estrita. Nota-se que o grau de indiferença (I) não seja necessariamente transitivo, enquanto o de preferência (P) ainda é transitivo. Suponha-se três alternativas $a_{1}, a_{2}$ e $a_{3}$, temos $a_{1} I a_{2}$ e $a_{2} I a_{3}$, porém $a_{1} P a_{3}$, conforme é apresentado na figura abaixo:

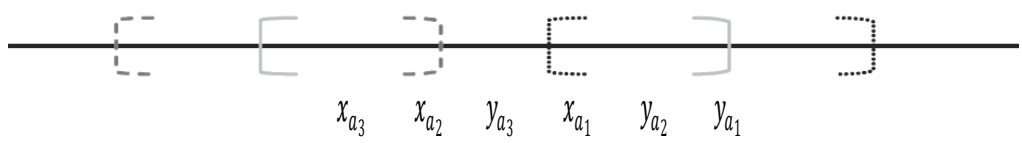

Figura 1 - Relações de a1, a2 e a3.

\section{ESTUDO DE CASO}

As vantagens proporcionadas pelas ARPs para aplicação na guerra naval, como minimização da exposição de aeronaves convencionais junto a sua tripulação e custos reduzidos de aquisição, operação e manutenção, estimularam a Marinha brasileira na análise dessa tecnologia para uma possível aquisição e emprego.

Dentre muitas variáveis e especificações para análise, a Marinha do Brasil deve seguir os Requisitos Operacionais Conjunto, pré-definidos pelo Ministério da Defesa. Visando o emprego das ARPs nas operações navais, o órgão militar possui um conjunto de aeronaves em avaliação, tabela 4, e estes equipamentos atendem aos requisitos necessários, entretanto por haver trade-off entre os critérios, será realizado uma análise multicriterial objetivando obter o equipamento mais favorável. 
Tabela 4 - Aeronaves Remotamente Pilotadas.

\begin{tabular}{|cc|}
\hline Empresa & Nome da ARP \\
\hline Ael & Hermes 900 \\
\hline Saab & Skeldar V-200 \\
\hline Schiebel & Camcopter s-100 \\
\hline Cassidian & Tanan 300 \\
\hline Indra & Pelicano \\
\hline Insitu & Scan Eagle \\
\hline
\end{tabular}

Havendo a presença de critérios com diferentes naturezas, a modelagem híbrida PROMETHEE-SAPEVO-M1, viabilizará uma análise decisória com dados de origem qualitativa e quantitativa. 0 método também permitirá a Marinha do Brasil obter, de forma estruturada, os pesos e importâncias respectivas a cada critério.

Para aplicação do método, foi desenvolvido uma ferramenta em linguagem PYTHON 3.7, que auxiliará o agente decisor em todo o processo de análise e avaliação, sendo desde a estruturação do caso até a obtenção dos resultados.

Considerando que os custos de aquisição da tecnologia estão ligados diretamente ao sistema RPAS como um todo, este critério não será analisado, considerando que o mesmo pode diferenciar de forma significativa por estar relacionado principalmente ao número de aeronaves presentes no sistema. Assim, serão apenas analisadas as aeronaves em si, avaliando estritamente os critérios de influência quanto ao desempenho operacional.

As ARPs avaliadas são pertencentes a classe estratégica, destinadas a operações de ataque, guerra eletrônica, enlace de dados, apoio ao combate, vigilância, sensoriamento e reconhecimento.

\subsection{ANÁLISE DO CASO PELA MODELAGEM PROMETHEE-SAPEVO-M1}

Para análise de decisão, foi considerado um conjunto de critérios composto por quatorze elementos. Os critérios avaliados estão ligados ao desempenho operacional de uma ARP, apresentado na figura 2 , os mesmos estão listados na tabela 5 , sendo especificado se este é de natureza qualitativa ou quantitativa e os dados referentes a cada aeronave dentro de cada critério. Todos os dados apresentados foram obtidos nos sites pertencentes a cada empresa responsável pelas ARPs analisadas. 


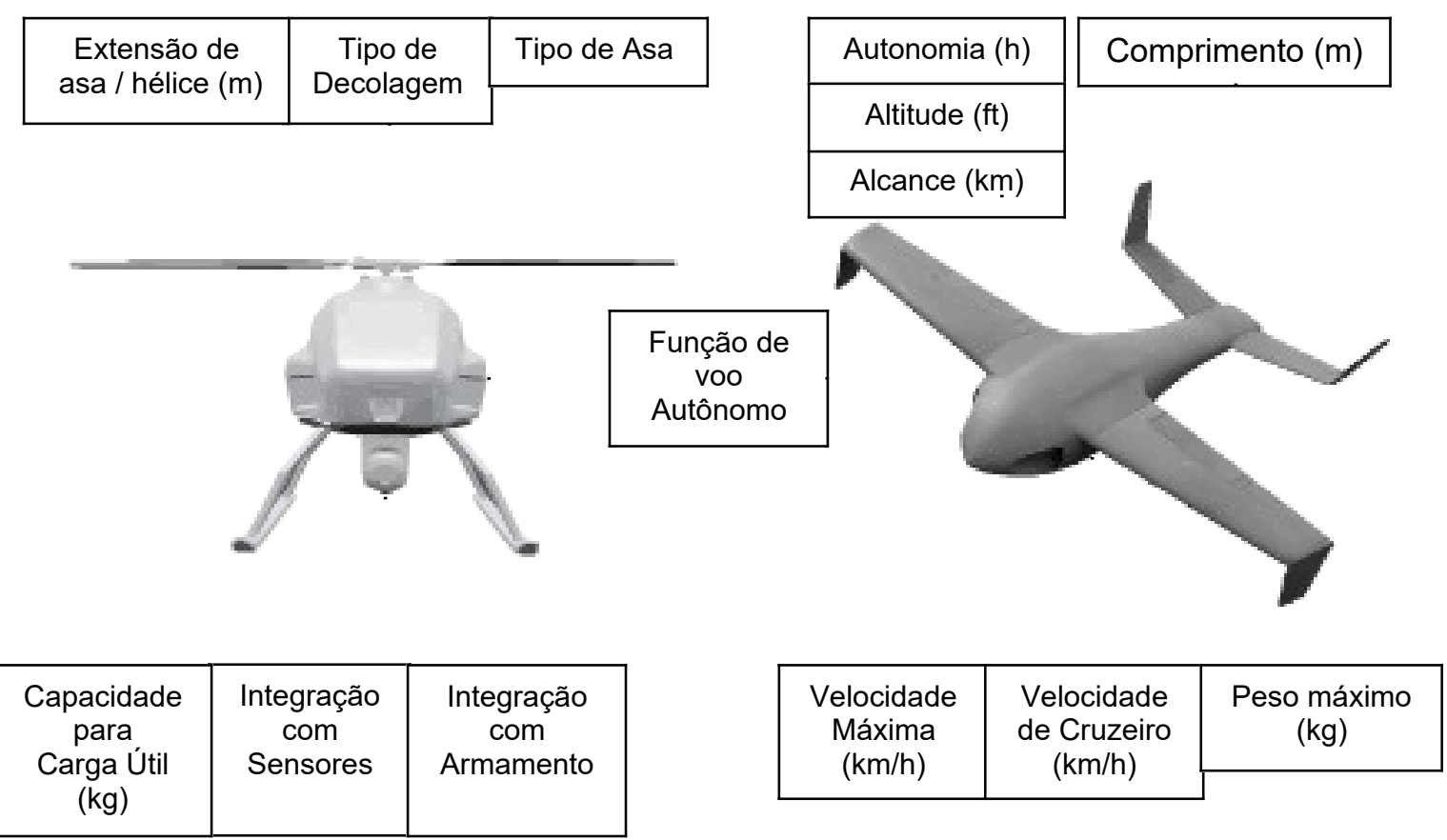

Figura 2 - Drones e seus critérios de avaliação.

Tabela 5 - Matriz de Avaliação para ARPs.

\begin{tabular}{|c|c|c|c|c|c|c|c|}
\hline & \multicolumn{6}{|c|}{ ALTERNATIVAS } \\
\hline & & $\begin{array}{c}\text { Hermes } \\
900\end{array}$ & $\begin{array}{l}\text { Skeldar } \\
\text { V-200 M }\end{array}$ & $\begin{array}{c}\text { Camcopter } \\
\text { S-100 }\end{array}$ & $\begin{array}{c}\text { Tanan } \\
\mathbf{3 0 0} \\
\end{array}$ & Pelicano & Scan Eagle \\
\hline \multirow{5}{*}{ 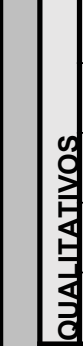 } & Tipo de Asa & Fixa & Rotativa & Rotativa & Rotativa & Rotativa & Fixa \\
\hline & Tipo de Decolagem & Livre (Pista) & Livre (base) & Livre (base) & Livre (base) & Livre (base) & Catapulta \\
\hline & Integração com Sensores & $\operatorname{sim}$ & $\operatorname{sim}$ & $\operatorname{sim}$ & $\operatorname{sim}$ & $\operatorname{sim}$ & $\operatorname{sim}$ \\
\hline & Integração com Armamento & não & não & $\operatorname{sim}$ & $\operatorname{sim}$ & $\operatorname{sim}$ & não \\
\hline & Função de voo Autônomo & $\operatorname{sim}$ & $\operatorname{sim}$ & $\operatorname{sim}$ & não & $\operatorname{sim}$ & não \\
\hline \multirow{9}{*}{ 氙最 } & Velocidade Máxima (Km/h) & 220 & 150 & 222 & 150 & 185 & 148 \\
\hline & Velocidade de Cruzeiro $(\mathrm{Km} / \mathrm{h})$ & 112 & 100 & 102 & 100 & 90 & 110 \\
\hline & Alcance $(\mathrm{Km})$ & Ilimitado & 200 & 200 & 180 & 100 & 100 \\
\hline & Autonomia (h) & 36 & 5 & 6 & 8 & 6 & 24 \\
\hline & Altitude (ft) & 30000 & 9842 & 18000 & 13000 & 11800 & 19500 \\
\hline & Peso máximo de decolagem $(\mathrm{Kg})$ & 1180 & 235 & 200 & 350 & 200 & 22 \\
\hline & Extensão de asa / hélice (m) & 15 & 4,6 & 3,4 & 6,3 & 3,3 & 3,11 \\
\hline & Comprimento (m) & 8,3 & 4 & 3,11 & 5,2 & 4 & 1,71 \\
\hline & Capacidade de Carga útil (Kg) & 350 & 40 & 50 & 80 & 30 & 3,4 \\
\hline
\end{tabular}


Ao se iniciar a ferramenta desenvolvida, a tela inicial faz uma breve descrição das etapas pertencentes ao método, e é destinada a estruturação do dado caso no software, como é exposto na figura 3, sendo requisitado as alternativas e critérios.

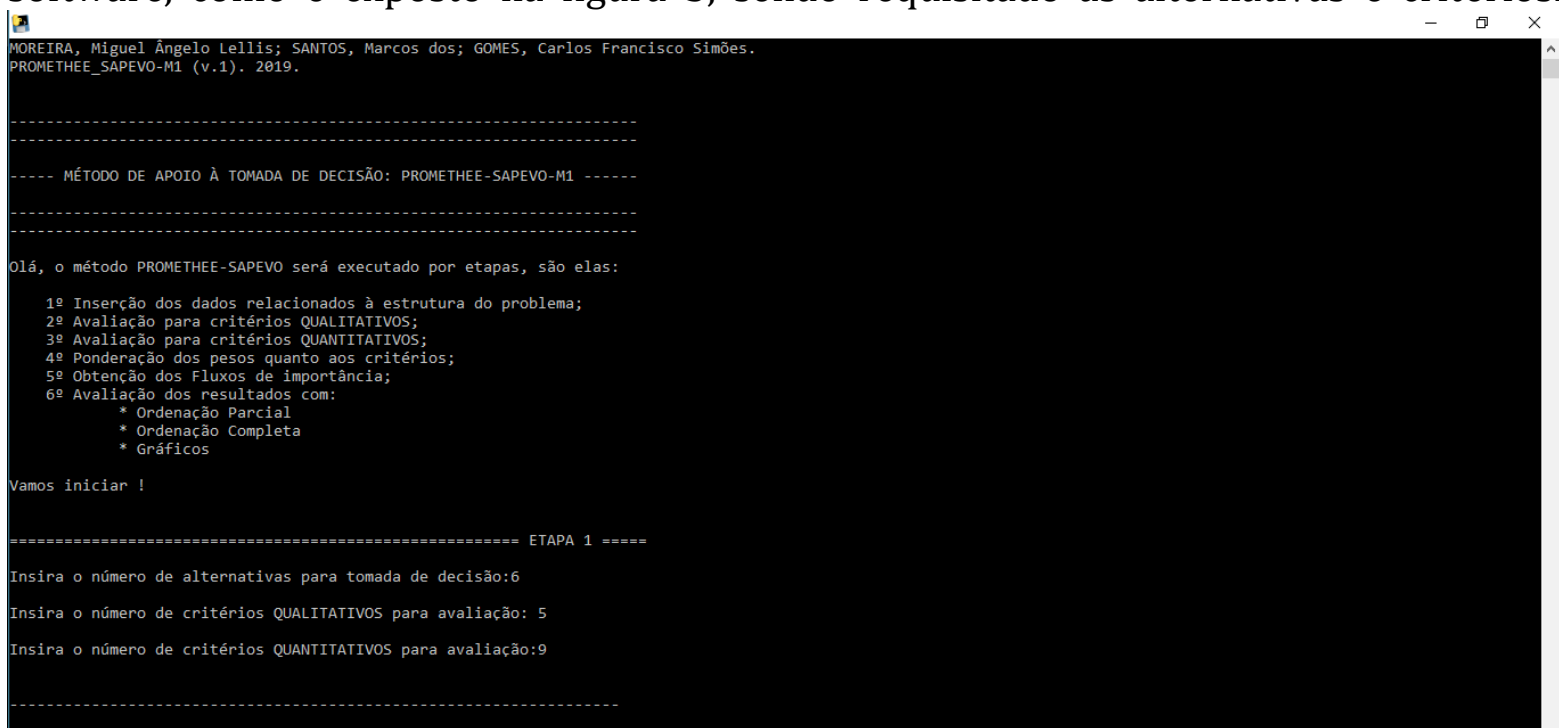

Figura 3 - Etapa Inicial do Software.

O software segue a mesma sequência lógica da modelagem proposta, sendo a primeira avaliação destinada aos critérios qualitativos, explorado no item 3.1.2. As alternativas são avaliadas par a par em todos os critérios de natureza qualitativa, ao final da avaliação é gerado uma matriz normalizada com os graus de preferência para cada alternativa no critério específico, conforme a figura 4.

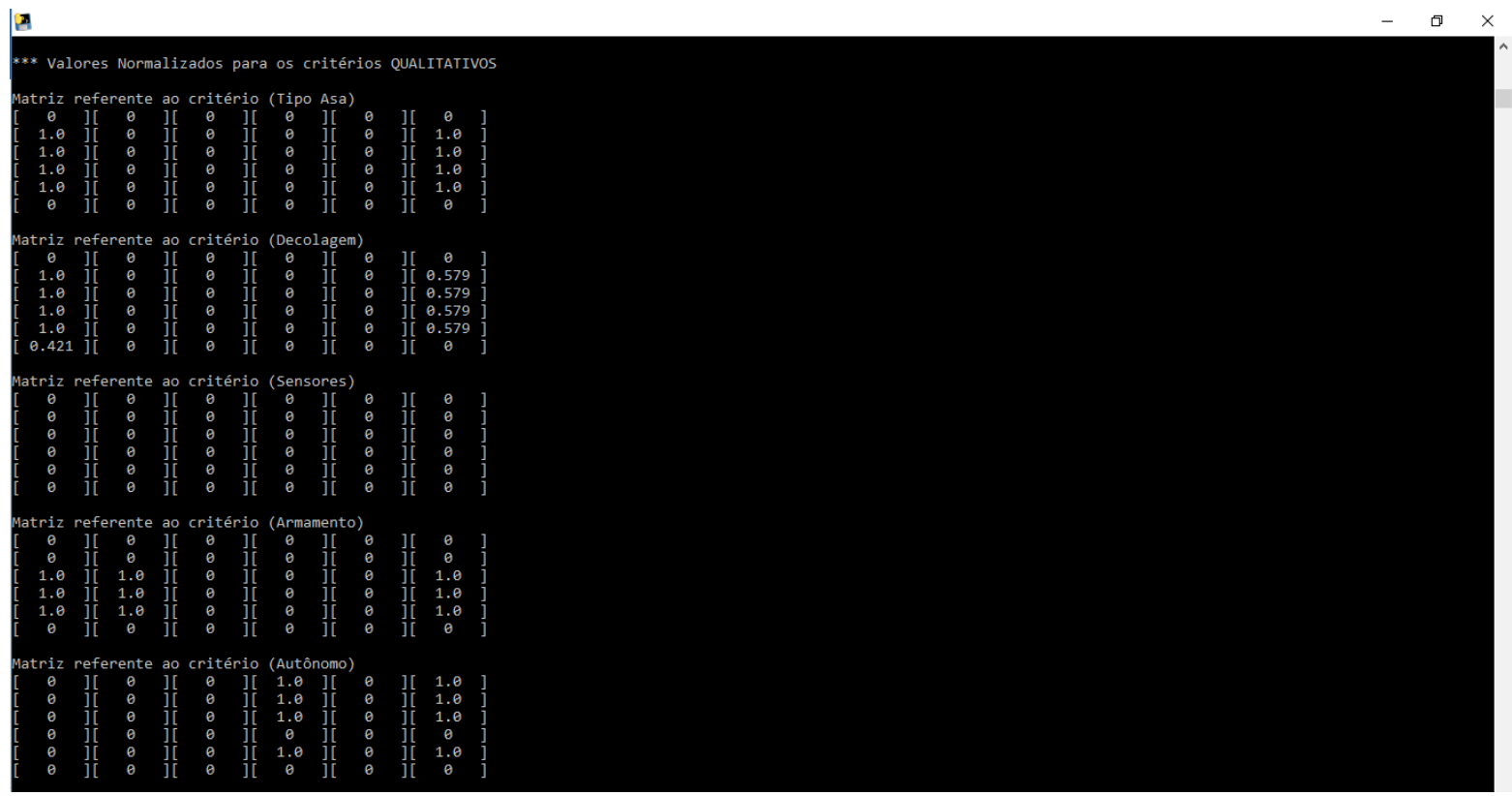

Figura 4 - Alternativas Normalizadas nos Critérios Qualitativos. 
Conforme exposto no item 3.1.3 o método segue realizando uma avaliação quantitativa, nela é solicitado ao decisor os valores numéricos respectivos a cada alternativa dentro de cada critério. Com os dados inseridos, o software solicita especificar quais dos critérios quantitativos necessitam serem maximizados ou minimizados e também é requerido indicar o tipo de função a ser utilizada para normalização junto aos seus parâmetros, a tabela 6 expõem essas informações.

\section{Tabela 6 - Funções de Avaliação Quantitativa}

\begin{tabular}{|c|c|c|c|c|c|}
\hline & & Funcão & Tino de Funcão & Par & tros \\
\hline & & runçao & I Ipo de Funçao & $q$ & $\mathbf{p}$ \\
\hline & Velocidade Máxima $(\mathrm{Km} / \mathrm{h})$ & Maximizar & V-Shape & -- & 30 \\
\hline$\stackrel{0}{\geq}$ & Velocidade de Cruzeiro (Km/h) & Maximizar & V-Shape & -- & 20 \\
\hline$\stackrel{5}{ \pm}$ & Alcance $(\mathrm{Km})$ & Maximizar & V-Shape & -- & 50 \\
\hline 至 & Autonomia (h) & Maximizar & V-Shape I & 1 & 2 \\
\hline$\stackrel{\text { DO }}{2}$ & Altitude (ft) & Maximizar & V-Shape I & 1000 & 3000 \\
\hline c & Peso máximo de decolagem $(\mathrm{Kg})$ & Minimizar & V-Shape & -- & 50 \\
\hline 은 & Extensão de asa / hélice (m) & Minimizar & V-Shape I & 0,5 & 1 \\
\hline 芒 & Comprimento (m) & Minimizar & V-Shape I & 1 & 2 \\
\hline & Capacidade de Carga útil $(\mathrm{Kg})$ & Maximizar & V-Shape & -- & 30 \\
\hline
\end{tabular}

A etapa seguinte é destinada a obtenção dos pesos, representando a importância de cada critério dentro do dado caso. Conforme apresentado no item 3.1.4 é feito uma avaliação par a par, a matriz obtida é exposta na tabela 7.

Tabela 7 - Avaliação de Comparação entre Critérios

\begin{tabular}{|c|c|c|c|c|c|c|c|c|c|c|c|c|c|c|}
\hline ESCALA DE COMPARAÇÃO & \multirow{8}{*}{ 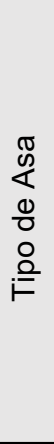 } & \multirow{8}{*}{$\begin{array}{l}\frac{\varepsilon}{0} \\
0 \\
\frac{\pi}{0} \\
0 \\
0 \\
0 \\
0 \\
0 \\
\circ \\
\stackrel{0}{=} \\
i=\end{array}$} & \multirow{8}{*}{ 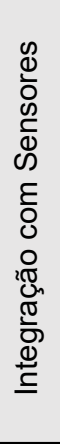 } & \multirow{8}{*}{ 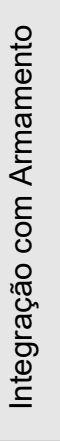 } & \multirow{8}{*}{ 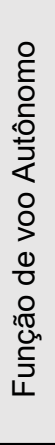 } & \multirow{8}{*}{ 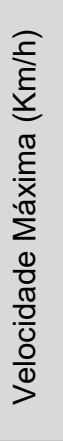 } & \multirow{8}{*}{ 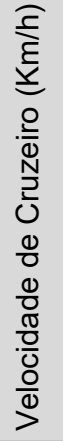 } & \multirow{8}{*}{ 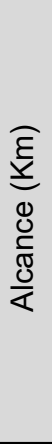 } & \multirow{8}{*}{ 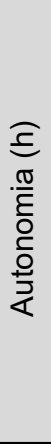 } & \multirow{8}{*}{ 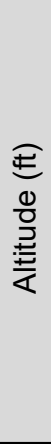 } & \multirow{8}{*}{ 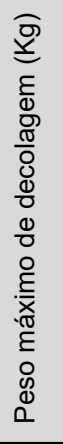 } & \multirow{8}{*}{ 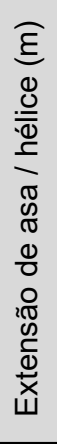 } & \multirow{8}{*}{ 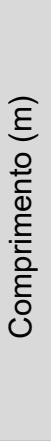 } & \multirow{8}{*}{ 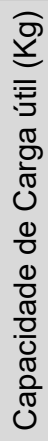 } \\
\hline Absolutamente pior $\quad-\mathbf{3}$ & & & & & & & & & & & & & & \\
\hline Muito Pior & & & & & & & & & & & & & & \\
\hline Pior & & & & & & & & & & & & & & \\
\hline Equivalente & & & & & & & & & & & & & & \\
\hline Melhor & & & & & & & & & & & & & & \\
\hline Muito melhor & & & & & & & & & & & & & & \\
\hline Absolutamente melhor $\mathbf{3}$ & & & & & & & & & & & & & & \\
\hline Tipo de Asa & 0 & -3 & -3 & -3 & -3 & -2 & -2 & -3 & -3 & -3 & -1 & 0 & 0 & -3 \\
\hline Tipo de Decolagem & 3 & 0 & 1 & 1 & 0 & 1 & 1 & 0 & 2 & 0 & 3 & 3 & 3 & 1 \\
\hline Integração com Sensores & 3 & -1 & 0 & 1 & 1 & 3 & 3 & 3 & 3 & 3 & 3 & 3 & 3 & 2 \\
\hline Integração com Armamento & 3 & -1 & -1 & 0 & -2 & 1 & 1 & -1 & 1 & 1 & 2 & 3 & 3 & 2 \\
\hline Função de voo Autônomo & 3 & 0 & -1 & 2 & 0 & 3 & 3 & 3 & 3 & 3 & 3 & 3 & 3 & 2 \\
\hline Velocidade Máxima $(\mathrm{Km} / \mathrm{h})$ & 2 & -1 & -3 & -1 & -3 & 0 & -2 & -1 & -1 & -1 & 0 & 2 & 2 & -2 \\
\hline Velocidade de Cruzeiro (Km/h) & 2 & -1 & -3 & -1 & -3 & 2 & 0 & 1 & 0 & 1 & 2 & 3 & 3 & 0 \\
\hline Alcance $(\mathrm{Km})$ & 3 & 0 & -3 & 1 & -3 & 1 & -1 & 0 & 0 & 0 & 3 & 3 & 3 & 1 \\
\hline Autonomia (h) & 3 & -2 & -3 & -1 & -3 & 1 & 0 & 0 & 0 & 0 & 1 & 2 & 2 & -1 \\
\hline Altitude (ft) & 3 & 0 & -3 & -1 & -3 & 1 & -1 & 0 & 0 & 0 & 1 & 2 & 2 & -1 \\
\hline Peso máximo de decolagem $(\mathrm{Kg})$ & 1 & -3 & -3 & -2 & -3 & 0 & -2 & -3 & -1 & -1 & 0 & 0 & 0 & -2 \\
\hline Extensão de asa / hélice (m) & 0 & -3 & -3 & -3 & -3 & -2 & -3 & -3 & -2 & -2 & 0 & 0 & 0 & -3 \\
\hline Comprimento (m) & 0 & -3 & -3 & -3 & -3 & -2 & -3 & -3 & -2 & -2 & 0 & 0 & 0 & -3 \\
\hline Capacidade de Carga útil $(\mathrm{Kg})$ & 3 & -1 & -2 & -2 & -2 & 2 & 0 & 1 & 1 & 1 & 2 & 3 & 3 & 0 \\
\hline
\end{tabular}


Os pesos obtidos em cada critério são apresentados a seguir:

Tabela 8 - Pesos dos Critérios.

\begin{tabular}{|l|l|}
\hline Tipo de Asa & $\mathbf{0 , 0 1 8}$ \\
\hline Tipo de Decolagem & $\mathbf{0 , 1 0 6}$ \\
\hline Integração com Sensores & $\mathbf{0 , 1 2 6}$ \\
\hline Integração com Armamento & $\mathbf{0 , 0 9 3}$ \\
\hline Função de voo Autônomo & $\mathbf{0 , 1 2 6}$ \\
\hline Velocidade Máxima $(\mathrm{Km} / \mathrm{h})$ & $\mathbf{0 , 0 5 5}$ \\
\hline Velocidade de Cruzeiro $(\mathrm{Km} / \mathrm{h})$ & $\mathbf{0 , 0 8 2}$ \\
\hline Alcance $(\mathrm{Km})$ & $\mathbf{0 , 0 8 6}$ \\
\hline Autonomia $(\mathrm{h})$ & $\mathbf{0 , 0 7 0}$ \\
\hline Altitude $(\mathrm{ft})$ & $\mathbf{0 , 0 7 1}$ \\
\hline Peso máximo de decolagem $(\mathrm{Kg})$ & $\mathbf{0 , 0 3 7}$ \\
\hline Extensão de asa / hélice $(\mathrm{m})$ & $\mathbf{0 , 0 2 2}$ \\
\hline Comprimento $(\mathrm{m})$ & $\mathbf{0 , 0 2 2}$ \\
\hline Capacidade de Carga útil $(\mathrm{Kg})$ & $\mathbf{0 , 0 8 4}$ \\
\hline
\end{tabular}

Com os pesos gerados, é calculado os índices de preferência ponderada global, neles é possível analisar o grau de dominância de cada ARP sobre as demais, par a par, e dentro do conjunto como um todo por meio dos fluxos de importância positivo, negativo e líquido. Na tabela 9 é apresentado os valores dos fluxos respectivos a cada alternativa.

Tabela 9 - Fluxos de Preferência.

\begin{tabular}{|cccc|}
\hline ARP & Fluxo Positivo & Fluxo Negativo & Fluxo Líquido \\
\hline Hermes 900 & 0.162 & 0.088 & 0.074 \\
\hline Skeldar V-200 M & 0.068 & 0.106 & -0.038 \\
\hline Camcopter s-100 & 0.129 & 0.048 & 0.081 \\
\hline Tanan 300 & 0.101 & 0.117 & -0.016 \\
\hline Pelicano & 0.084 & 0.107 & -0.023 \\
\hline Scan Eagle & 0.078 & 0.156 & -0.078 \\
\hline
\end{tabular}

\subsection{ANÁLISE DOS RESULTADOS}

Com os fluxos gerados é possível obter as avaliações de preferência parcial, total e por intervalos. A primeira análise, figura 5, apresenta uma avaliação pelos fluxos positivos, coluna direita no gráfico, e negativos, coluna esquerda no gráfico, quanto maior o fluxo positivo e menor o negativo da alternativa, melhor é a opção.

No caso trabalhado, as opções mais favoráveis foram as ARPs Camcopter S-100 e Hermes 900, onde entre si obtiveram relação de incomparabilidade, e entre as demais, ambas são preferíveis. As aeronaves Skeldar V-200M, Pelicano e Tanan 300, obtiveram relação de incomparabilidade entre si e as duas últimas registraram graus de preferência quanto a ARP Scan Eagle, que dentro do contexto apresentado não foi preferível a nenhuma opção. 


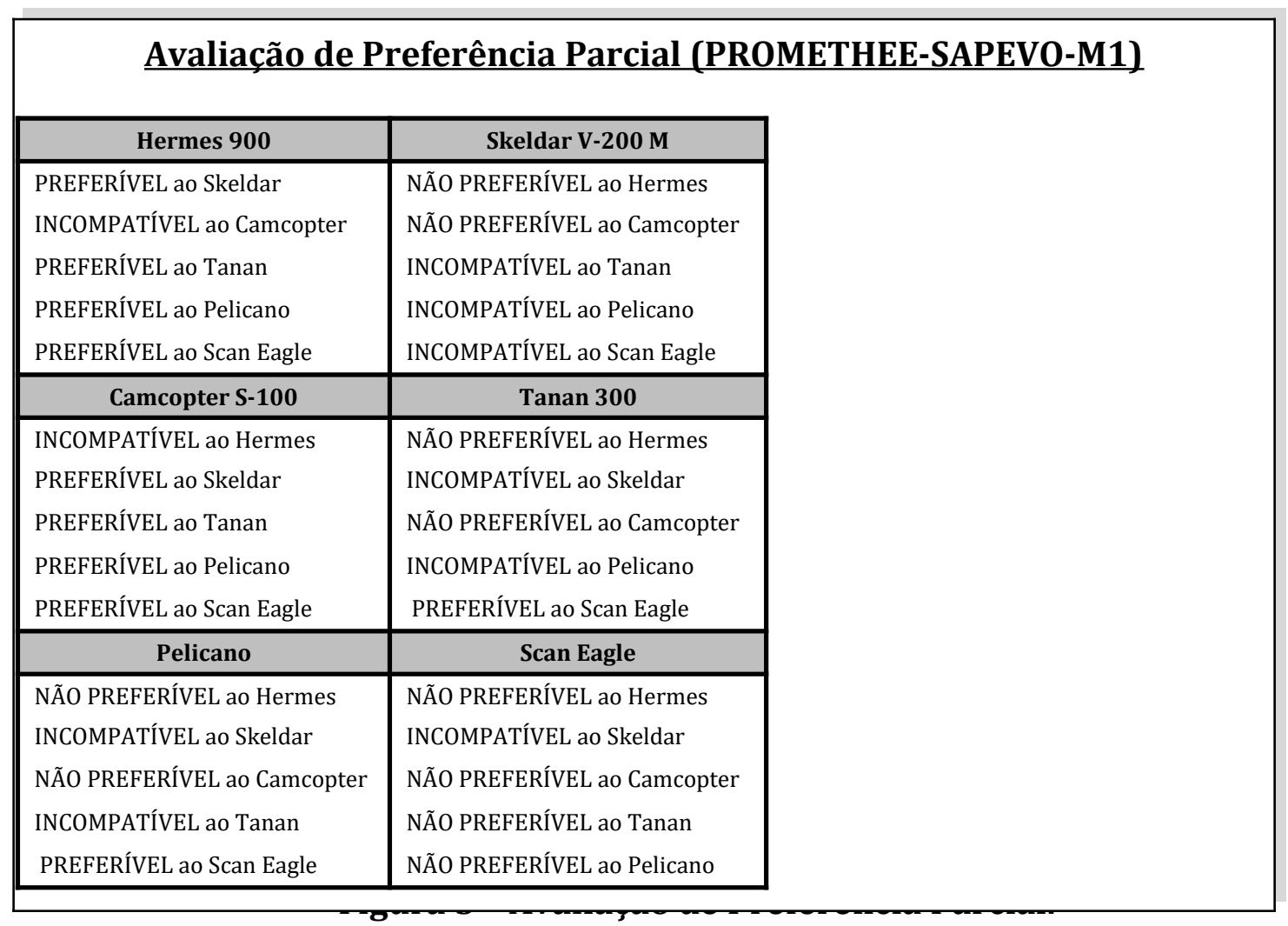

A segunda análise, avaliação de preferência total, é apresentada uma ordenação de preferência da alternativa mais favorável ao caso até a menos favorável. A análise é gerada através dos fluxos de importância líquidos, quanto maior o valor obtido, melhor é a opção. No estudo de caso as ARPs Camcopter S-100 e Hermes 900 obtiveram os melhores resultados, entretendo a primeira apresentou uma pequena vantagem em relação a segunda, podendo ser classificada como a opção mais favorável.

Os resultados gerados pelo software são expostos na figura 6, sendo analisado a ordenação obtida junto com uma apresentação gráfica do resultado.

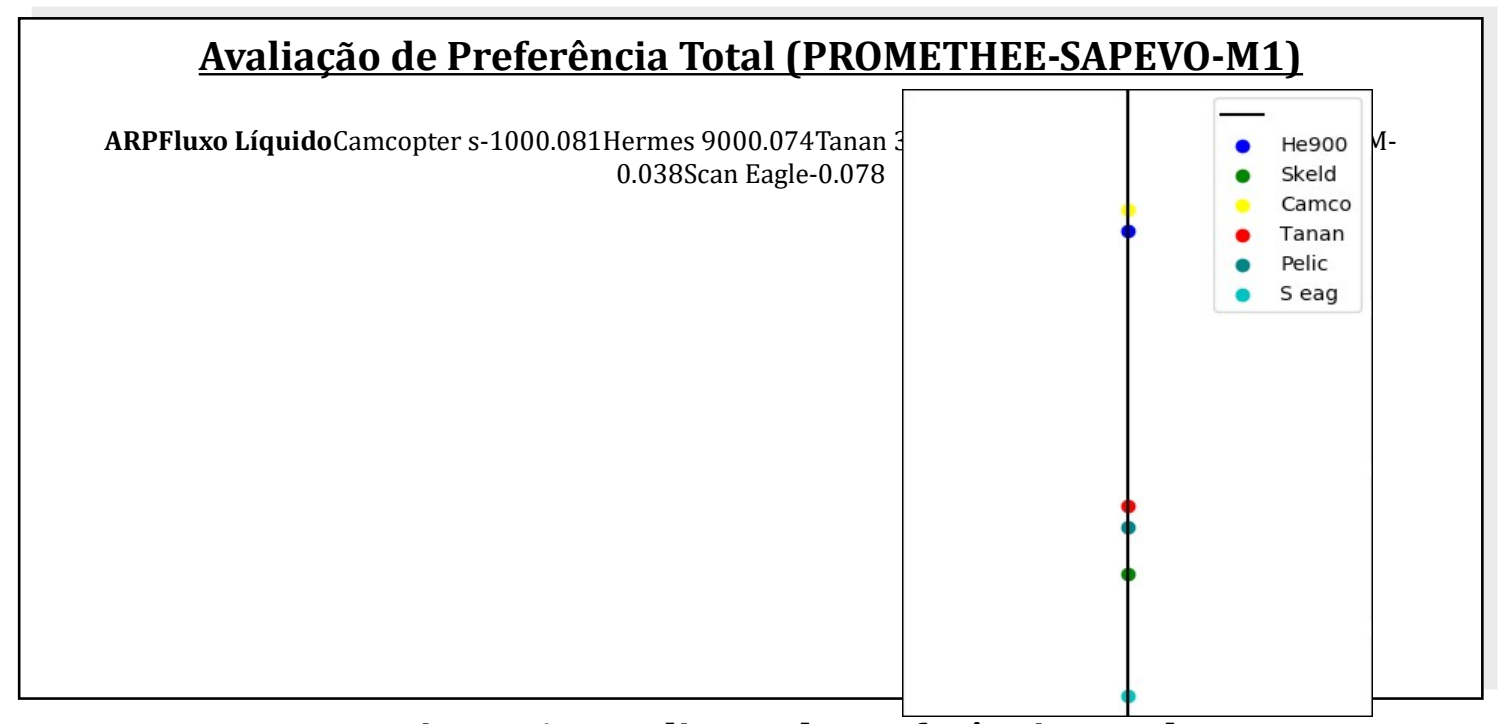

Figura 6 - Avaliação de Preferência Total. 
O último modelo de análise, avaliação de preferência por intervalos, inclui uma avaliação onde por meio de um valor de erro padrão, obtido pela amostra de resultados do fluxo líquido, é possível gerar índices superiores e inferiores, caso um dos índices de uma alternativa cruze com o de outra, a relação de preferência entre elas passa a ser de indiferença, caso contrário é exposto se a relação é de preferência ou não preferência.

No contexto estudado, as opções com melhores avaliação, Camcopter S-100 e Hermes 900, se tornam indiferentes uma a outra e ambas se tornam preferíveis em relação as demais alternativas do conjunto. Uma grande vantagem deste modelo de análise é saber se realmente uma alternativa é superior a outra. As informações geradas pela ferramenta operada são expostas na figura abaixo.

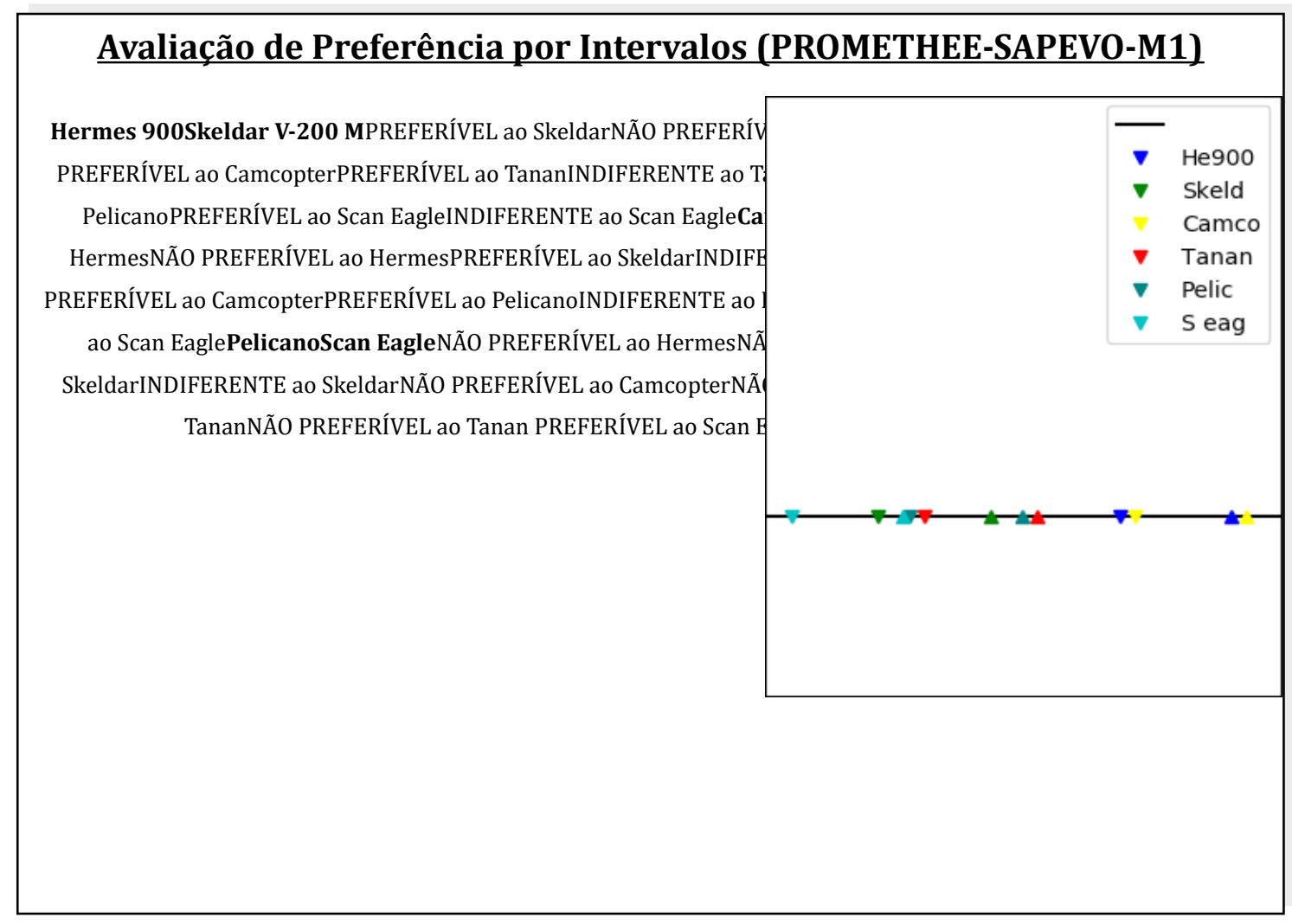

Figura 7 - Avaliação de Preferência Total.

Os resultados apresentados são expostos no script da ferramenta e os gráficos são expostos de forma conjunto ao final da execução, o modelo pode ser salvo no desktop do usuário. A apresentação conjunta dos gráficos, entrega ao agente decisor uma análise de sensibilidade do problema, onde é possível saber o quão melhor uma alternativa pode ser sobre a outra ou não, permitindo aos valores obtidos nos fluxos, serem analisados sob três formas diferentes de avaliação.

Além dos gráficos, as avaliações de dominância entre as alternativas podem ser avaliadas em cada critério, qualitativo ou quantitativo, por meio das matrizes que são impressas no script da ferramenta. Todo o processo lógico matemático da modelagem é exposto na ferramenta, podendo ser analisado em todas as etapas as relações apresentadas entre cada par de alternativas. 


\section{CONSIDERAÇÕES FINAIS}

O estudo desenvolvido teve por objetivo, estar apresentando um caso real em que a aplicação de um método de Apoio Multicritério à Decisão viabilizou uma avaliação de alto impacto para a Marinha do Brasil.

0 novo método híbrido apresentado, PROMETHEE-SAPEVO-M1, mostra sua eficiência e eficácia, a partir do momento em que habilita operar dados de diferentes naturezas de forma equivalente quanto as suas análises e resultados. A nova proposta apresenta sua robustez entregando uma obtenção de pesos de forma estruturada e uma análise de resultados baseada em três modelos de ordenação de preferência.

A ferramenta desenvolvida em linguagem PYTHON, oferece significativos ganhos quanto sua forma de aplicação, guiando o agente decisor durante todo o processo de inserção de dados e avaliação das alternativas quanto aos critérios. 0 software apresentado pode ser aplicado em diferentes contextos de análise decisória com múltiplos critérios de avaliação.

Quanto a Marinha do Brasil, espera-se este trabalho apoiar o órgão militar em uma tomada de decisão relacionada ao reconhecimento de quais ARPs são mais favoráveis em uma aplicação eficiente e eficaz em suas operações na guerra naval.

Como proposta para trabalhos futuros, pretende-se estar aplicando o método PROMETHEE-SAPEVO-M1 em outros casos de análise decisória multicriterial, buscando identificar pontos de melhorias na modelagem ou novas técnicas a serem integradas, garantindo maior robustez ao novo método de apoio à tomada de decisão com múltiplos critérios.

\section{REFERÊNCIAS BIBLIOGRÁFICAS}

[1] BRAGA, Cláudio da Costa. A Ação de Drones na Guerra Naval. Revista Marítima Brasileira, v. 139. Rio de Janeiro, 2019.

[2] BRANS, J.; VINCKE, Ph. A preference ranking organization method: The PROMETHEE method for MCDM. Management Science, v. 31, n. 6, p. 647656, 1985.

[3] BRANS, Jean-Pierre; DE SMET, Yves. PROMETHEE methods. In: Multiple criteria decision analysis. Springer, New York, NY, 2016. p. 187-219.

[4] BRANS, Jean-Pierre; VINCKE, Ph; MARESCHAL, Bertrand. How to select and how to rank projects: The PROMETHEE method. European journal of operational research, v. 24, n. 2, p. 228-238, 1986.

[5] BRASIL. Marinha do Brasil. Estado Maior da Armada. EMA-305: Doutrina Básica da Marinha. Brasília, DF, 2014

[6] BRASIL. Comando da Aeronáutica. Departamento de Controle do Espaço Aéreo. Trafego Aéreo. AERONAVES NÃO TRIPULADAS E O ACESSO AO ESPAÇO AÉREO BRASILEIRO, 2018. Disponível em: https://www.decea.gov.br/drone/. Acesso em: Março, 2019.

[7] FLOREANO, Dario; WOOD, Robert J. Science, technology and the future of small autonomous drones. Nature, v. 521, n. 7553, p. 460, 2015.

[8] GIONES, Ferran; BREM, Alexander. From toys to tools: The co-evolution of technological and entrepreneurial developments in the drone industry. Business Horizons, v. 60, n. 6, p. 875-884, 2017.

[9] Gomes, L. F. A. M., e Gomes, C. F. S. Princípios e métodos para a tomada de decisão: Enfoque multicritério (6a ed.). São Paulo: Atlas. (2019). 
[10] GOMES, L. F. A. M.; MURY, Antonio R.; GOMES, Carlos FS. Multicriteria ranking with ordinal data. Systems Analysis Modelling Simulation, v. 27, p. 139-146, 1997.

[11] GRECO, Salvatore; FIGUEIRA, José; EHRGOTT, Matthias. Multiple criteria decision analysis. New York: Springer, 2016.

[12] PECHARROMÁN, José M. P.; VEIGA, Ricardo. Estudo Sobre a Indústria Brasileira e Europeia de Veículos Aéreos Não Tripulados. [S. l.], 2016. Disponível em: http://www.mdic.gov.br/images/publicacao_DRONES-20161130-20012017-web.pdf. Acesso em: Março, 2019.

[13] SILVA, Leandro de Oliveira. APLICAÇÃo DE VANTS NA LOGÍSTICA HUMANITÁRIA: PROCEDIMENTO PARA DISTRIBUIÇÃO E ROTEAMENTO DE VEÍCULOS. Dissertação de Mestrado em Engenharia de Transportes, Instituto Militar de Engenharia, Rio de Janeiro, 2016.

[14] SILVA, Moisés Câmara. A" revolução militar" dos drones (2001 a 2018): da" caçada humana" no Afeganistão às várias frentes de batalha no Oriente Médio e o aumento da escala da guerra entre as" grandes potências". Dissertação de Mestrado em Relações Internacionais, Universidade Estadual da Paraíba, João Pessoa, 2018.

[15] TZENG, Gwo-Hshiung; HUANG, Jih-Jeng. Multiple attribute decision making: methods and applications. Chapman and Hall/CRC, 2011. 